ISSN: 0210-1696

DOI: http://dx.doi.org/10.14201/scero20154637383

\title{
A3BYCOMP: UNA HERRAMIENTA DE APOYO PARA LA IMPLANTACIÓN BÁSICA DE SISTEMAS DE GESTIÓN POR COMPETENCIAS EN ENTIDADES SOCIALES
}

\section{A3byComp: A Support tool for Basic Implementation of Competency Management Systems in Social Entities}

\author{
Daniel Clavero Herrero \\ daniel.clavero@claveropersson.com
}

Recepción: 19 de junio de 2015

Fecha de aceptación definitiva: 22 de julio de 2015

Biblid. [0210-1696 (2015) vol. 46 (3), n. ${ }^{\circ}$ 255, julio-septiembre; 73-83]

Resumen: La importancia de la gestión de los procesos relacionados con las personas que trabajan en las entidades pertenecientes al ámbito de FEAPS se evidencia en la creciente incorporación a la estrategia de las organizaciones de políticas orientadas a la mejora de las condiciones laborales de los trabajadores, así como en el desarrollo de sistemas de gestión por competencias orientados a la promoción del desarrollo profesional, cuya finalidad es una mayor eficacia y eficiencia en la prestación de apoyos para la mejora de la calidad de vida de las personas con discapacidad y sus familias. Al mismo tiempo, las entidades han puesto de manifiesto la necesidad de contar con instrumentos y herramientas específicas que faciliten la implantación de estos sistemas. En Extremadura, se ha desarrollado una herramienta informática para la implantación de sistemas básicos de gestión por competencias denominada A3byComp que se ha puesto gratuitamente a disposición de todo el sector promoviendo una red de colaboración cuyo objetivo es el aprendizaje mutuo.

Palabras Clave: gestión por competencias; plan de personas; calidad de vida; desarrollo profesional; FEAPS.

AвstRACT: The importance of managing processes related to people working in institutions within the scope of FEAPS evidenced by the increasing incorporation into the strategy of the organizations policies aimed at improving the working conditions of workers and in the development of competence management systems aimed at promoting professional development, which aims greater effectiveness and efficiency in providing 
support for improving the quality of life of people with disabilities and their families. At the same time, banks have highlighted the need for specific instruments and tools that facilitate the implementation of these systems. In Extremadura, it has been developed a software tool for the implementation of basic competence management systems called A3byComp that has been freely available to the entire network promoting industry collaboration aimed mutual learning.

KEY WORDS: management skills; people plan; quality of life; professional development; FEAPS

\section{Introducción}

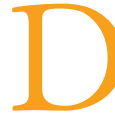

ESDE UN PUNTO DE VISTA PURAMENTE ESTRATÉGICO, la gestión de recursos humanos alineada con el cumplimiento de la misión de cualquier organización supone grandes beneficios y es un elemento generador de ventajas competitivas que pueden llegar a ser predictoras de éxito en las organizaciones (Burke, 2005; Strandskov, 2006; Gooderham et al., 2008).

Según FEAPS (2007), el Proyecto LIDER (Liderar, Integrar, Desarrollar y Reconocer) tiene como objetivo que el conjunto del Movimiento Asociativo desarrolle Planes de Personas coherentes con su Modelo de Política de Personas. Responde a una nueva orientación en la dirección y desarrollo de personas en nuestras organizaciones, considerando éstas como un factor crítico y diferencial de las mismas.

En relación a la gestión por competencias, López (2010) considera que ésta aporta un valor mayor que la gestión tradicional por tareas o, más recientemente, la dirección por objetivos ya que, mientras que la gestión por tareas se centra en lo que se tiene que hacer y la dirección por objetivos en lo que se ha de alcanzar, la gestión por competencias integra y supera los anteriores modelos centrando su atención en la conducta humana y las competencias de las personas que condicionan su comportamiento.

Con respecto al marco laboral que afecta a las organizaciones, el 9 de octubre de 2012 el Ministerio de Empleo y Seguridad Social publicaba en el Boletín Oficial del Estado núm. 243 el XIV Convenio colectivo general de centros y servicios de atención a personas con discapacidad introduciendo una gran novedad con respecto a los anteriores convenios del sector: el concepto de desarrollo profesional basado en competencias.

Dada la creciente importancia y necesidad de contar con herramientas que favorezcan la implantación de sistemas de gestión de recursos humanos por competencias alineados con los paradigmas aceptados en el sector, a continuación se describe el marco teórico y las características de una de ellas, A3byComp, desarrollada por el autor y puesta a disposición gratuitamente de todas las entidades pertenecientes a FEAPS.

\section{Marco teórico de referencia}

El inicio del siglo XX fue el escenario del surgimiento de las competencias como un nuevo enfoque del éxito de una organización, pero no es hasta la década de los 70 cuando en Estados Unidos adquiere mayor importancia su estudio. 
A continuación vamos a hacer referencia a algunas de las definiciones más importantes que, desde su origen, se han realizado sobre el concepto de competencia.

El concepto "Competencia” fue introducido por McClelland (1973) cuando en su artículo "Pruebas de competencia en lugar de inteligencia” apuntó como elemento predictor del éxito en el trabajo a características de las personas relacionadas con los conocimientos, habilidades, rasgos de la personalidad, etc., en lugar de los tradicionales test psicológicos que hasta la fecha eran utilizados en exclusiva.

Con posterioridad, Boyatzis (1982) define las competencias como características subyacentes en una persona, que están causalmente relacionadas con una actuación exitosa en un puesto de trabajo.

Más recientemente Spencer y Spencer (1993) defienden que la competencia es una característica subyacente en el individuo que está causalmente relacionada a un estándar de efectividad y/o a una performance superior en un trabajo o situación.

Goleman (1998) por su parte considera que una competencia es un rasgo personal o un conjunto de hábitos que llevan a un desempeño laboral superior o más eficaz o, por decirlo de otro modo, una habilidad que aumenta el valor económico del esfuerzo que una persona realiza en el mundo laboral.

De estas definiciones y especialmente de la propuesta por Spencer y Spencer (1993) podemos destacar tres elementos principalmente:

A) La competencia como característica subyacente en el individuo

De acuerdo con Spencer y Spencer (1993), las competencias son ante todo características fundamentales del hombre e indican formas de comportamiento o de pensar, que generalizan diferentes situaciones y duran por un largo período de tiempo, pudiéndose clasificar estas en 5 tipos (motivación, rasgos de personalidad, autocomprensión, conocimientos y habilidades).

Esto último queda reflejado en el Modelo de Iceberg propuesto por los autores (Figura 1).

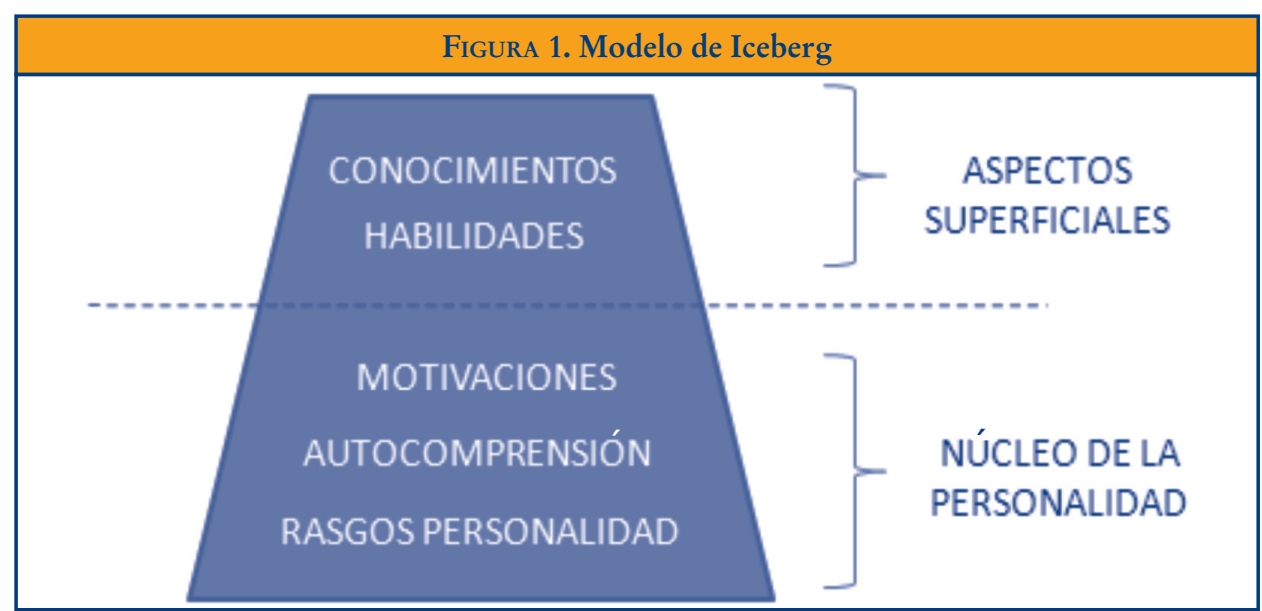

Fuente: Adaptado de Spencer y Spencer (1993). 
B) La competencia como relación causal con el comportamiento

Según Spencer y Spencer (1993), las competencias siempre incluyen una intención que es el motivo o la fuerza del rasgo que causa la acción hacia un resultado, por lo que la conducta sin intención no define una competencia. Por tanto, las relaciones causales entre las características fundamentales de la persona, ya sean desde sus aspectos más centrales (como la motivación, rasgos de la personalidad y la autocomprensión) así como desde aquellas más visibles (como el conocimiento y las habilidades), se deben evidenciar en los resultados que producen los comportamientos derivados de ellas.

De esta manera, se puede establecer una relación causal entre la motivación, el comportamiento derivado de ella y los resultados obtenidos (Figura 2).

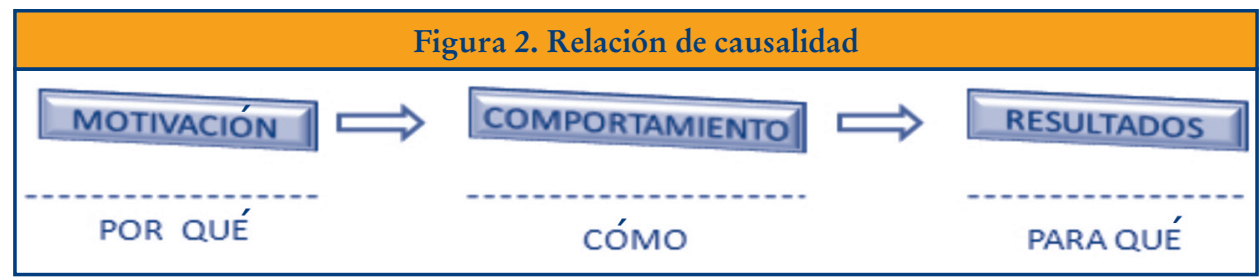

Fuente: Adaptado de Spencer y Spencer (1993).

C) La competencia como elemento predictor basado en estándares

Siguiendo a Spencer y Spencer (1993), considerándose las competencias como características de las personas, éstas no se podrán considerar una competencia a no ser que sean un elemento predictor de algo significativo en relación al rendimiento o desempeño. En relación a esto Spencer y Spencer (1993) señalan que los criterios más habituales en las competencias son: a) Rendimiento Superior: identificado como un porcentaje de incremento sobre unos valores estándar asociado a una situación de trabajo concreta, y b) Rendimiento Eficaz: referido a un mínimo aceptable de nivel de trabajo por debajo del cual un trabajador se considera incompetente.

Una vez realizado un acercamiento al concepto de competencia, a continuación, vamos a determinar un marco de referencia que nos permita establecer un punto de origen para el desarrollo de sistemas de gestión por competencias en FEAPS.

En relación a la implantación de sistemas de gestión por competencias, según Alles (2007), es necesario empezar por la definición de la visión de la empresa, sus objetivos y la misión para, a partir de ello, decidir cómo llevarlo a cabo a través del diseño e implantación del modelo en los diferentes procesos de recursos humanos.

En ese sentido es de gran interés el Modelo FEAPS de Política de Personas que consiste en un enfoque de política integral de desarrollo de personas y se estructura en base a un alineamiento entre las bases corporativas de FEAPS (calidad de vida de las personas con discapacidad intelectual y sus familias, calidad en la gestión, ética y 
valores, y otras prioridades contingentes) y las bases funcionales del propio modelo (liderar, integrar, desarrollar y reconocer), configurando políticas clave que se orientan a la satisfacción personal y profesional de los trabajadores y voluntarios del Movimiento Asociativo, y al cumplimiento de la misión de las organizaciones.

Nos encontramos, por tanto, en el ámbito de FEAPS con un marco teórico que puede servir de base para relacionar casualmente la motivación, el comportamiento y la orientación a resultados que, desde una perspectiva competencial, deben caracterizar a las personas que trabajen en el sector.

Basándonos en definición de Spencer y Spencer (1993) y partiendo de la estructura del Modelo FEAPS de Política de Personas, enmarcado dentro del Sistema de Calidad FEAPS, podemos establecer un marco de referencia para el establecimiento de un Sistema de Gestión por Competencias (Figura 3).

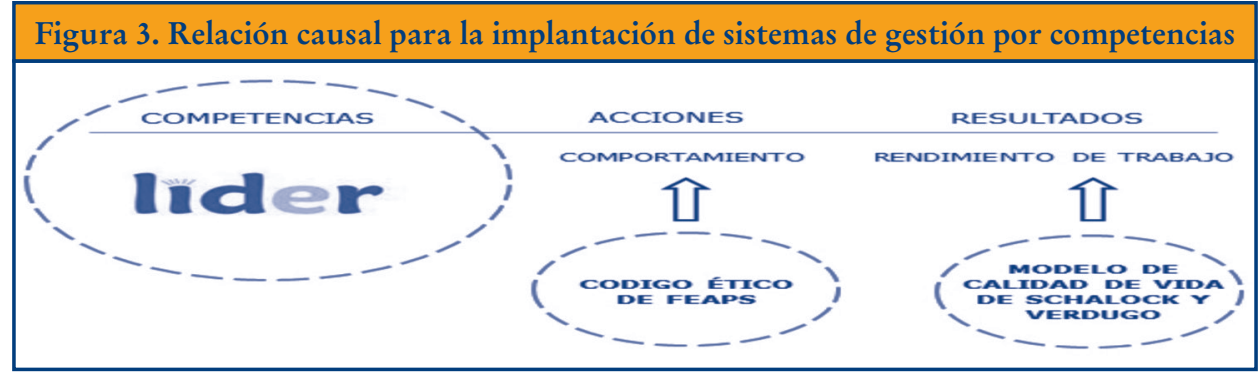

Fuente: Elaboración propia.

Por tanto, las competencias que debe promover la Política de Personas y caracterizar a los profesionales del sector deben evidenciarse a través de comportamientos coherentes con el Código Ético de FEAPS y materializarse en resultados personales medidos en términos de mejora de las diferentes dimensiones de calidad de vida de las personas con discapacidad según el Modelo de Calidad de Vida de Schalock y Verdugo (2003).

De esta manera, un primer acercamiento a la clasificación de las competencias se puede establecer de acuerdo a la siguiente matriz competencial (Tabla 1).

\begin{tabular}{|l|l|l|l|l|l|l|}
\hline \multicolumn{6}{|c|}{ Tabla 1. Propuesta de clasificación de Competencias en el ámbito de FEAPS } \\
\hline \multicolumn{1}{|c|}{ Competencias } & Calidad de vida & Ética & Gestión & \multicolumn{2}{c|}{ Ámbito } \\
\hline Conocimientos & & & & Genéricas & Específicas \\
\hline Habilidades & & & & & \\
\hline Motivaciones & & & & & \\
\hline Autocomprensión & & & & & \\
\hline Rasgos de personalidad & & & & & \\
\hline
\end{tabular}

Fuente: Elaboración propia. 
En esta matriz se establece una clasificación de competencias en base a las características subyacentes de los individuos (Spencer y Spencer, 1993) alineadas con las bases corporativas del Modelo de Política de Personas de FEAPS, estableciendo un nivel genérico u organizativo de aplicación y un nivel específico o de puesto.

Por otra parte, el XIV Convenio colectivo en el desarrollo de sus artículos 87, 88 y 89 regula los siguientes aspectos de vital importancia a nivel laboral en nuestras entidades:

a) Establece un nuevo sistema de mejora de competencias profesionales basada en grupos profesionales, niveles y tramos de cualificación.

b) Regula los mecanismos de evaluación que permitirán acceder a un nivel superior de cualificación y al reconocimiento salarial del complemento de desarrollo profesional que corresponda (y que sustituye al antiguo concepto de antigüedad).

c) Determina un sistema de trabajo en red, a través de la Comisión Paritaria que sirve de órgano interpretativo de dicho convenio, para apoyar a las entidades en el desarrollo de este nuevo modelo y para centralizar el conocimiento que se genere en el sector en relación a la implantación y desarrollo del mismo.

d) Describe una primera clasificación de competencias generales que deben caracterizar a los diferentes perfiles profesionales.

Podemos afirmar que el espíritu del nuevo convenio colectivo es orientar a las entidades en la introducción de sistemas de gestión de recursos humanos por competencias, haciendo especial atención a la evaluación del desempeño, motivo por el que se hace aún más necesario el estudio y desarrollo de un marco de referencia acorde a la misión de las organizaciones del sector, evitando simplemente el mero cumplimiento de la normativa laboral.

Las entidades adheridas al convenio laboral al que hemos hecho referencia y en especial aquellas que se incorporaron al sistema antes del 31 de diciembre de $2013 \mathrm{y}$, por tanto, se comprometieron a realizar una evaluación de competencias antes del 31 de diciembre de 2015, han expresado su deseo de contar con estudios y herramientas que faciliten a las organizaciones el cambio de modelo que conlleva este nuevo marco laboral, motivo por el cual A3byComp se ofreció gratuitamente a aquellas organizaciones interesadas con el compromiso de participar en una red profesional que facilitara el intercambio de experiencias en torno a la gestión por competencias y la mejora continua de la herramienta informática.

A continuación vamos a describir las principales características de la aplicación A3byComp.

\section{Descripción de la herramienta A3byComp}

A3byComp es una herramienta informática desarrollada para facilitar la implantación de sistemas básicos de gestión por competencias en entidades sociales. 
Su estructura de funcionamiento se basa en la definición de competencia formulada por Spencer y Spencer (1993) y más concretamente en la relación de causalidad que debe existir entre las competencias y los resultados esperados derivados de los comportamientos asociados a ellas, en nuestro caso, resultados medidos en términos de mejora de la calidad de vida de los usuarios y las familias destinatarias de los apoyos que prestan las entidades sociales en sus centros y servicios.

Es relación a esto, la herramienta propone una definición de los puestos de trabajo existentes en la entidad en base a dos perfiles diferenciados: un perfil funcional en el que se detallan las tareas que se deben realizar en virtud del puesto y un perfil competencial en el que se recogen las competencias idóneas para el desempeño eficaz de dichas tareas, partiendo de la hipótesis de que existe una relación causal entre ambos perfiles.

Para la construcción de ambos perfiles, A3byComp facilita, por una parte, el diseño de un catálogo de competencias y de comportamientos asociados a ellas (Figura 4), en función de los distintos niveles de desempeño y, por otra parte, el diseño de las tareas, clasificadas por funciones, que caracterizan el desarrollo de la práctica laboral de cada puesto de trabajo.

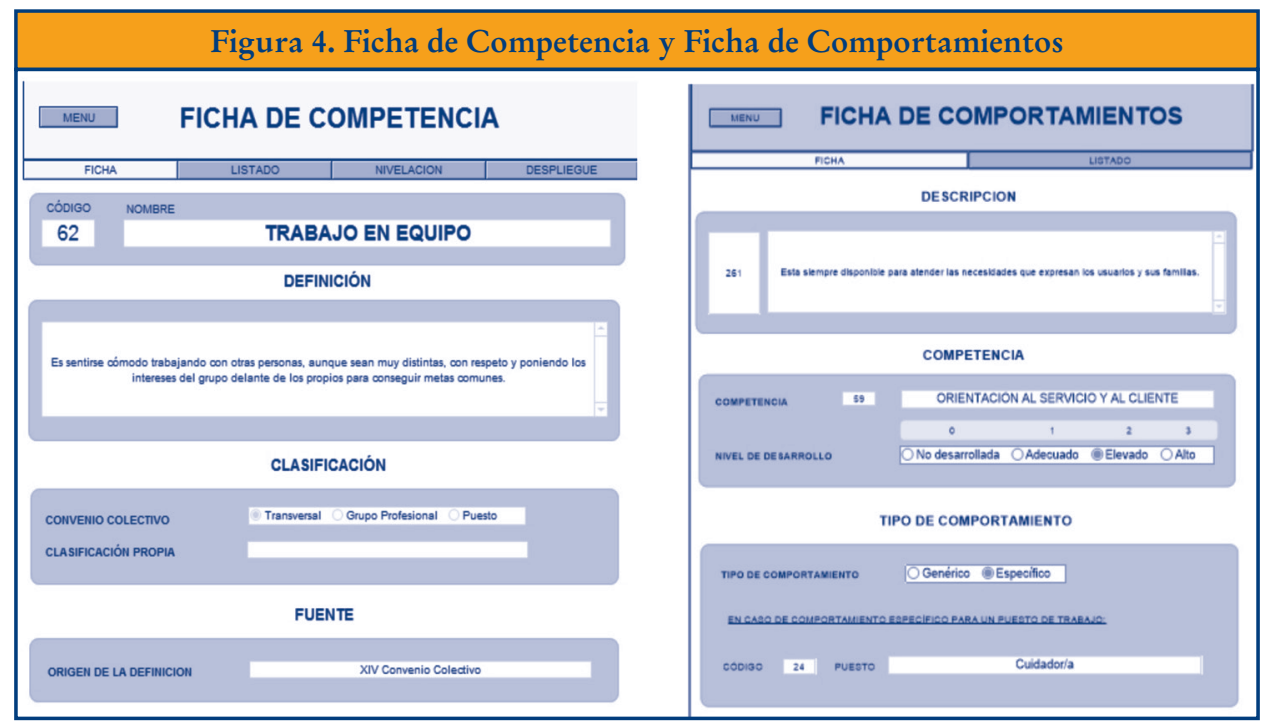

En relación a los comportamientos asociados a las competencias, A3byComp permite su descripción y clasificación por niveles de desempeño (no desarrollada, adecuado, elevado y alto) así como su asociación con carácter general o específico a un puesto de trabajo, lo cual permite informar con carácter específico como se evidencia la existencia de dicha competencia en el desempeño laboral de un puesto en concreto.

Para el diseño de las tareas que conforman el perfil funcional del puesto A3byComp no solo se limita a su definición sino que propone su alineamiento con las bases 
corporativas del modelo FEAPS de Política de Personas, acorde al marco del sistema de Calidad FEAPS.

Este alineamiento consiste en un proceso de reflexión sobre el impacto de las tareas en la calidad de vida del usuario en base a las 8 dimensiones del modelo de calidad de vida de Schalock y Verdugo (2003), la calidad de gestión y la ética, lo cual permite integrar no sólo lo que debemos hacer sino también el cómo y para qué lo hacemos (Figura 5).

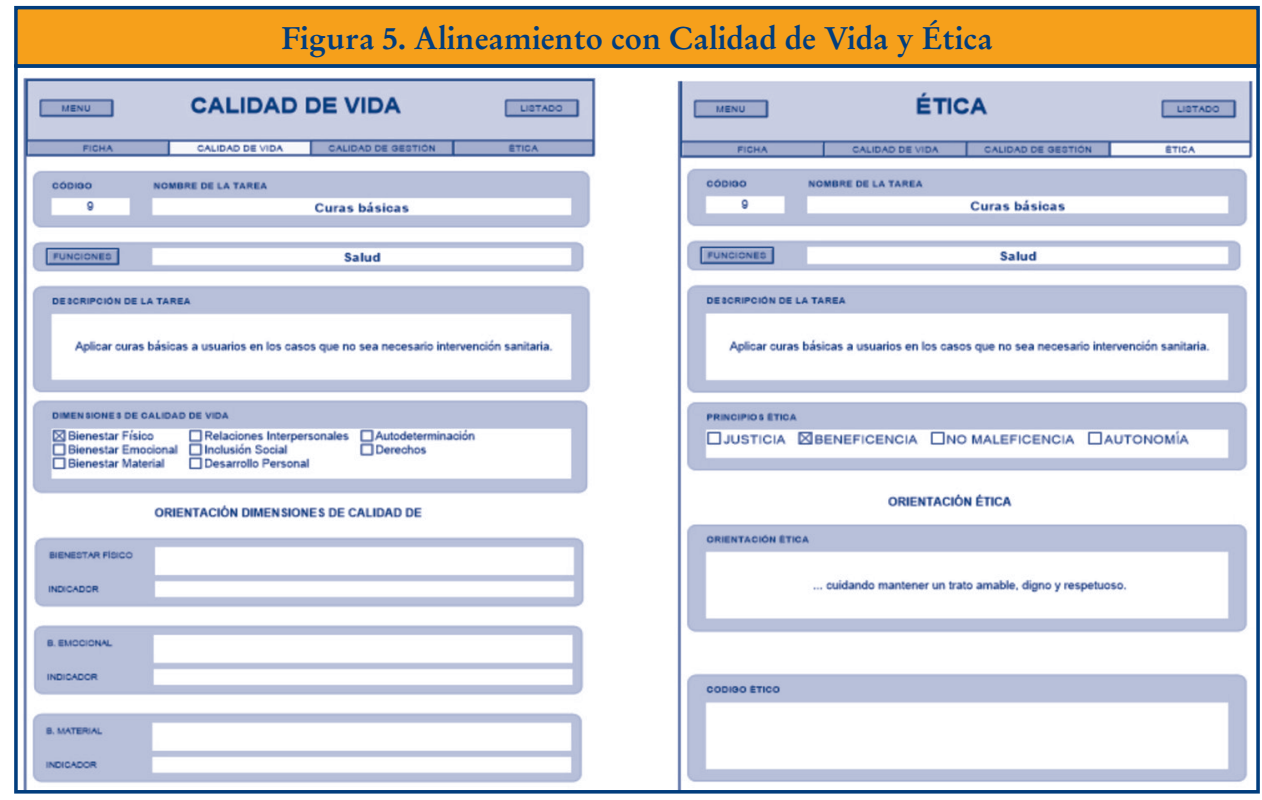

En lo referente a la construcción de los puestos de trabajo en base a su perfil funcional y competencial, A3byComp permite la ponderación de las competencias y tareas de cada perfil para que pueda informarse sobre la importancia de cada una de ellas dentro de su perfil, lo cual facilitará una mejor evaluación del desempeño a posteriori e identificación de áreas de mejora.

La aplicación permite también asignar a cada trabajador el puesto que ocupa, asociándose automáticamente el perfil competencial y funcional que corresponde a dicho puesto, y evaluar el grado de adecuación persona-puesto como punto de partida para establecer un plan de desarrollo profesional.

Para ello, A3byComp cuenta con un sistema de evaluación del desempeño $360^{\circ}$ basada en cuestionarios que puede ser configurado por la entidad para adaptarse a sus necesidades, en base a la importancia que se les otorgue a los 3 criterios en los que se basa: evaluadores (autoevaluación, pares y superior), perfil (competencial y funcional) y grado de adecuación al perfil exigido. 


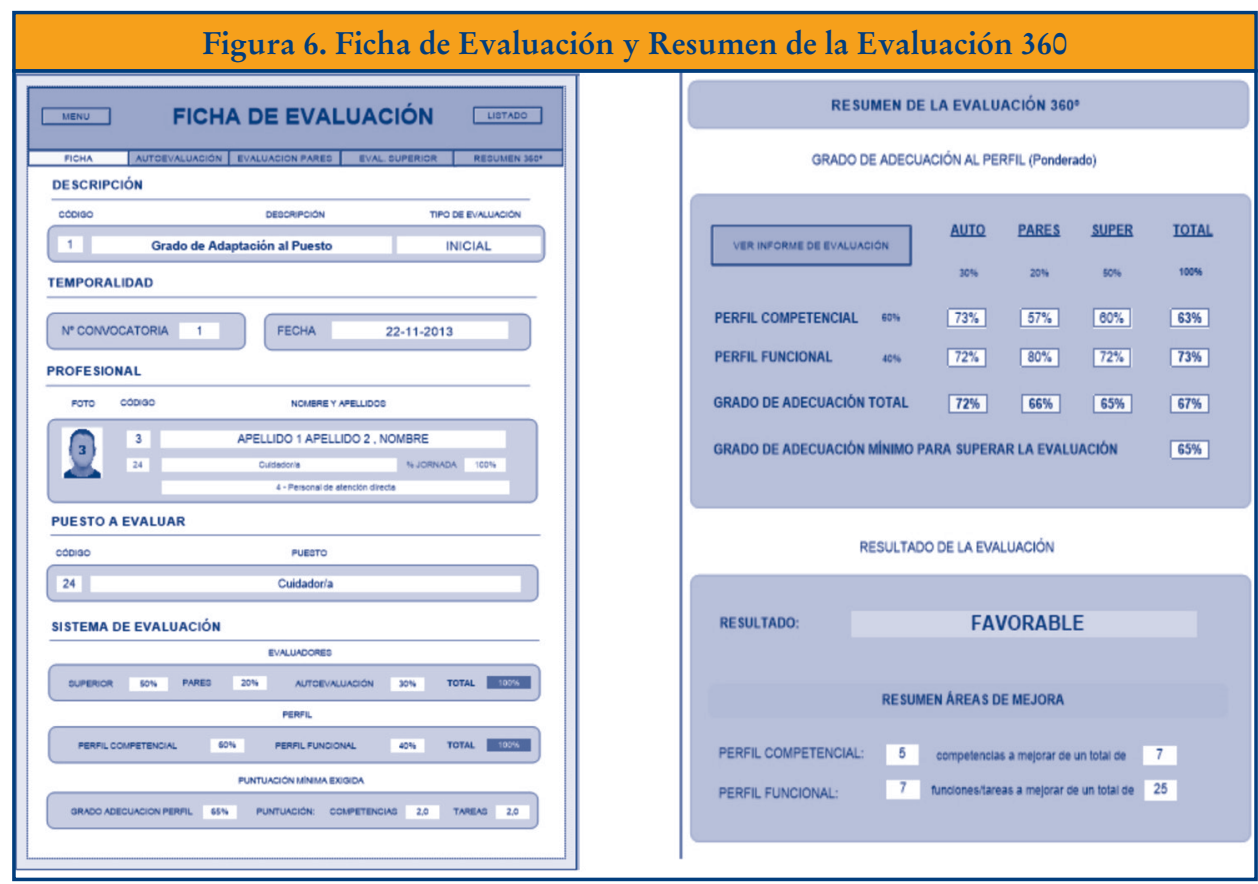

En relación a esto, la adaptación de estos parámetros a la situación en la que se encuentra la entidad permite que se establezcan unos criterios de referencia de rendimiento superior y rendimiento eficaz coherentes y orientados a la mejora continua.

Por último y en base a las evaluaciones realizadas, A3byComp permite la definición de planes de desarrollo profesional orientados a las áreas de mejora identificadas en la evaluación del perfil funcional y competencial del puesto ocupado, así como el diseño de recursos de formación de diferente naturaleza.

\section{Conclusiones}

A nivel técnico, A3byComp consiste únicamente en una herramienta de apoyo informático que debe ser acompañada de un proceso de reflexión interno en la entidad que permita identificar los contenidos relacionados con la identificación de competencias, comportamientos, funciones y tareas, así como su grado de alineamiento con la estrategia organizativa y los modelos comúnmente aceptados.

Es por ello que se recomienda, para el diseño del sistema de gestión por competencias que opere en la entidad, con un enfoque centrado en el cliente, una metodología que al menos cuente con los siguientes pasos:

a) Definición de misión, visión y valores de la organización.

b) Identificación del catálogo de puestos de trabajo existentes en la entidad. 
c) Realización de una encuesta o estudio para identificar y analizar las funciones (basada en áreas de apoyo a la persona) y tareas de cada puesto.

d) Desarrollo de grupos focales que permitan el alineamiento de las funciones y tareas con el modelo de Calidad de Vida de Schalock y Verdugo (2003).

e) Desarrollo de grupos focales que permitan un estudio ético del puesto para identificar comportamientos deseados en la práctica profesional y dar respuesta a dilemas éticos.

f) Desarrollo de grupos focales que permitan identificar las competencias generales y específicas, su nivelación, así como su categorización según las bases corporativas del modelo Feaps de Política de Personas, requeridas para el desarrollo de las funciones y tareas de cada puesto de trabajo.

g) Validación del diseño de cada puesto por los grupos de interés (profesionales, personas con discapacidad intelectual o del desarrollo y familiares).

h) Definición o adaptación de los diferentes subprocesos de gestión de recursos humanos (reclutamiento, selección, incorporación, desarrollo profesional y evaluación de desempeño) en relación a la definición de puestos de trabajo realizado basado en competencias.

Para terminar, y en relación a la responsabilidad social corporativa, El Libro Verde Fomentar un marco europeo para la responsabilidad social de las empresas (COM, 2001), promovido por el Consejo Europeo de Lisboa celebrado en 2000 y que fue publicado por la Comisión Europea en 2001, hace referencia a tres aspectos de vital importancia en relación con la gestión de los recursos humanos desde una perspectiva socialmente responsable:

a) En primer lugar, hace referencia a la atracción y mantenimiento de trabajadores cualificados, recomendando la puesta en marcha de medidas que fomenten el aprendizaje permanente, la responsabilización de los trabajadores, la mejora de la información en la empresa, un mayor equilibrio entre trabajo, familia y ocio, una mayor diversidad de recursos humanos, la igualdad de retribución y de perspectivas profesionales para las mujeres, la participación en los beneficios o en el accionariado de la empresa y la consideración de la capacidad de inserción profesional y la seguridad en el lugar de trabajo.

b) En segundo lugar, se propone la incorporación de prácticas responsables en los procesos de contratación de personal en las empresas, con el objetivo de facilitar la contratación de colectivos en riesgo de exclusión social como medida de lucha contra el desempleo y la discriminación social.

c) En tercer lugar, hace referencia al ámbito del aprendizaje permanente, a través de la promoción de un entorno que estimule el aprendizaje permanente de todos los trabajadores, especialmente de aquellos con más necesidades formativas.

El cumplimiento de las directrices anteriormente mencionadas es compatible con la cultura de aprendizaje que caracteriza los sistemas de gestión por competencias. 
Por tanto, el reto de las entidades sociales que trabajan en el sector de atención a personas con discapacidad en España no es otro que el de desarrollar sistemas de gestión por competencias socialmente responsables que sean eficaces y eficientes en la mejora de la calidad de vida de las personas con discapacidad y sus familias y, al mismo tiempo, fomenten una mejora de la calidad de vida laboral de los personas que trabajan en dichas entidades, entendida esta última como el fomento de las condiciones y un ambiente de trabajo favorable que proteja y promueva la satisfacción de los empleados mediante recompensas, seguridad laboral y oportunidad de desarrollo personal (Lau y May, 2000), como elemento sustancial de reconocimiento del desempeño laboral, y es un reto que estamos obligados moralmente a superar entre todos.

\section{Referencias bibliográficas}

Alles, M. A. (2007). Dirección estratégica de recursos humanos: gestión por competencias. Buenos Aires: Ediciones Granica, SA.

Boyatzis, R. E. y Royatzis, R. (1982). The competent manager: A model for effective performance. Wiley New York.

Burke, R. J. (2005). Reinventing Human Resource Management Challenges and New Directions, R. J. Burke, C. L. Cooper (Eds.). London: Routledge.

Comisión de las Comunidades Europeas, COM (2001). Libro Verde, Fomentar un Marco Europeo para la Responsabilidad Social de las Empresas, 366 - 18/VII/2001.

FEAPS (2007). Proyecto LIDER. Modelo FEAPS de Política de Personas. Edición Electrónica. URL: http://www.feaps.org/archivo/centro-documental/doc_download/363-modelo-feapsde-politica-de-personas.html. (Consulta 15 febrero 2013).

Goleman, D. (1998). Working with emotional intelligence. Bantam.

Gooderham, P., Parry, E. y Ringdal, K. (2008). The Impact of Bundles of Strategic Human Resource Management Practices on the Performance of European Firms. International Journal of Human Resource Management, 19 (11), 2041-2056.

López, E. R. (2010). Gestión por competencias. La Coruña: Netbiblo S. L.

LAU, R. y MAY, B. E. (1998). A win-win paradigm for quality of work life and business performance. Human Resource Development Quarterly, vol. 9, 3, 211-226.

McClelland, D. C. (1973). Testing for competence rather than for intelligence. American Psychologist, 28 (1), 1.

Resolución de 20 de septiembre de 2012, de la Dirección General de Empleo, por la que se registra y publica el XIV Convenio colectivo general de centros y servicios de atención a personas con discapacidad. Boletín Oficial del Estado, núm. 243 (9 de octubre de 2012).

Schalock, R. L. y Verdugo, M. Á. (2003). Calidad de vida: Manual para profesionales de la educación, salud y servicios sociales. Madrid: Alianza Editorial.

Spencer, L. y Spencer, S. (1993). Competency at work. New York: John Wiely \& Sons.

Spencer, L. y Spencer, S. (1993). Competency at work. New York: John Wiely \& Sons.

STRANDSKOv, J. (2006). Sources of competitive advantages and business performance. Journal of Business Economics and Management, 7 (3), 119-129. 